\title{
A precise HST parallax of the cataclysmic variable EX Hydrae, its system parameters, and accretion rate ${ }^{\star}$
}

\author{
K. Beuermann ${ }^{1}$, Th. E. Harrison ${ }^{2, * *}$, B. E. McArthur ${ }^{3}$, G. F. Benedict ${ }^{3}$, and B. T. Gänsicke ${ }^{4}$ \\ ${ }^{1}$ Universitäts-Sternwarte Göttingen, Geismarlandstr. 11, 37083 Göttingen, Germany \\ e-mail: beuermann@uni-sw.gwdg.de \\ 2 New Mexico State University, Box 30001/MSC 4500, Las Cruces, NM 88003 USA \\ e-mail: tharriso@nmsu.edu ${ }^{\star \star}$ \\ 3 McDonald Observatory, University of Texas, Austin, TX 78712 USA \\ e-mail: mca@barney.as.utexas.edu; fritz@astro.as.utexas.edu \\ ${ }^{4}$ Department of Physics and Astronomy, University of Southampton, Highfield, Southampton SO17 1BJ, UK \\ e-mail: btg@astro.soton.ac.uk
}

Received 26 March 2003 / Accepted 15 September 2003

\begin{abstract}
Using the HST Fine Guidance Sensor, we have measured a high precision astrometric parallax of the cataclysmic variable EX Hydrae, $\pi=15.50 \pm 0.29$ mas. From the wavelength-integrated accretion-induced energy flux, we derive a quiescent accretion luminosity for EX Hya of $L_{\mathrm{acc}}=(2.6 \pm 0.6) \times 10^{32} \mathrm{erg} \mathrm{s}^{-1}$. The quiescent accretion rate then is $\dot{M}_{\mathrm{av}}=(6.2 \pm 1.5) \times 10^{-11}\left(M_{1} / 0.5 M_{\odot}\right)^{-1.61} M_{\odot} \mathrm{yr}^{-1}$. The time-averaged accretion rate, which includes a small correction for the rare outbursts, is $6 \%$ higher. We discuss the system parameters of EX Hya and deduce $M_{1}=0.4-0.7 M_{\odot}, M_{2}=0.07-0.10 M_{\odot}$, and $i=76.0^{\circ}-77.6^{\circ}$, using recent radial velocity measurements of both components and restrictions imposed by other observational and theoretical constraints. We conclude that the secondary is undermassive, overluminous, and expanded over a ZAMS star of the same mass. Near the upper limit to $M_{1}$, the accretion rate of the white dwarf coincides with that due to nearequilibrium angular momentum loss by gravitational radiation and angular momentum transfer from the orbit into the spin-up of the white dwarf. Near the lower mass limit, the correspondingly higher accretion rate requires that either an additional angular momentum loss process is acting besides gravitational radiation or that accretion occurs on a near-adiabatic time scale. The latter possibility would imply that EX Hya is in a transient phase of high mass transfer and the associated spin-up of the white dwarf.
\end{abstract}

Key words. astrometry - stars: individual: EX Hydrae - stars: variables: general

\section{Introduction}

Cataclysmic variables (CVs) are ideal sites for the study of accretion processes. Understanding these phenomena requires the knowledge of distances and luminosities. Unfortunately, trigonometric parallaxes, the only secure source for distances, are so far available only for a handful of CVs (Harrison et al. 1999, 2000, 2003; McArthur et al. 1999, 2001). EX Hya is one of the best-studied intermediate polars, i.e. CVs in which the rotation of the magnetic white dwarf is not synchronized with

Send offprint requests to: K. Beuermann,

e-mail: beuermann@uni-sw.gwdg.de

* Based on observations made with the NASA/ESA Hubble Space Telescope, which is operated by the Association of Universities for Research in Astronomy, Inc., under NASA contract NAS 5-26555. These observations are associated with proposal \#9230.

$\star \star$ Visiting Astronomer, Cerro Tololo Inter-American Observatory, National Optical Astronomy Observatory, which is operated by the Association of Universities for Research in Astronomy, Inc., under cooperative agreement with the National Science Foundation. the orbital period. Accretion spins up the white dwarf on a time scale of $(3.8 \pm 0.2) \times 10^{6} \mathrm{yrs}$ (Hellier \& Sproats 1992).

Earlier distance estimates range from $105 \mathrm{pc}$ (Warner 1987) to $>130 \mathrm{pc}$ (Berriman et al. 1985), while the recent detection of the ellipsoidal light modulation of the secondary star in the infrared suggests a much smaller distance of $65 \mathrm{pc}$ (Eisenbart et al. 2002, henceforth EBRG02). Only a trigonometric distance will allow an accurate measurement of the luminosity and the accretion rate to be made. The latter is an essential ingredient for the discussion of the secular evolution of $\mathrm{CVs}$ and of the angular momentum loss processes driving accretion. Since EX Hya is not included in ground-based parallax programs, we have obtained a trigonometric parallax using the HST Fine Guidance Sensor (FGS).

\section{Observations and data reduction}

The process for deriving a parallax for a cataclysmic variable from FGS observations has been fully described in papers by McArthur et al. $(2001,1999)$ and Harrison et al. (1999). 
The process used here is identical to those efforts. An FGS program consists of a series of observations of the target of interest, and a set of four or more reference stars located close to that target. Typically, three epochs of observations, each comprised of two or more individual pointings (HST orbits), are used to solve for the variables in the series of equations that define a parallax solution. For EX Hya we were able to obtain observations on four different epochs (2000 July, 2000 December, 2001 July, and 2002 January). The extra epoch was fortuitous in that the reference frame for EX Hya was somewhat "noisy". As described in McArthur et al. (2001) extensive calibration data, as well as estimates of the distances and proper motions of the reference stars, are required to obtain a robust parallax solution.

\subsection{Spectroscopic parallaxes of the reference frame}

We have used a combination of spectroscopy and photometry to estimate spectroscopic parallaxes for the reference stars. Optical BVRI photometry was obtained on 2001 March 13 using the CTIO $0.9 \mathrm{~m}$ telescope and the Cassegrain Focus CCD imager $^{1}$. These data were reduced in the normal fashion, and calibrated to the standard system using observations of Landolt standards. The final photometric data set is listed in Table 1. Included in Table 1 is the Two Micron All-Sky Survey (2MASS) $J H K$ photometry of the reference stars, transformed to the homongenized system of Bessell \& Brett (1988) using the transformation equations from Carpenter (2001). Typical error bars on the photometry range from \pm 0.02 mag for the $V$-band measurements, to \pm 0.04 mag for the 2 MASS photometry of the fainter stars (except for EX-193, where the error on $B-V$ is $\pm 0.05 \mathrm{mag})$.

Optical spectroscopy of the reference frame stars, and a number of MK spectral type templates, was obtained on 2001 March 9 and 10 using the CTIO 1.5-m telescope with the Cassegrain Spectrograph ${ }^{2}$. We used the $831 \mathrm{l} / \mathrm{mm}$ "G-47" grating (resolution $0.56 \AA /$ pix) with a two arcsecond slit. From comparison of the spectra of the program objects to those of the MK-templates, we estimated the spectral types of the reference stars listed in Table 1.

By combining the spectral types of the reference frame stars with their photometry, we can derive the visual extinction to each target. Using the standard relations from Reike \& Lebofsky (1985), the final visual extinctions $A_{\mathrm{V}}$ were computed (see Table 2). Except for the most distant of the reference stars, the $A_{\mathrm{V}}$ values cluster near $0.20 \mathrm{mag}$. The interstellar hydrogen column density to EX Hya as measured by the Ly $\alpha$ absorption profile is only $3 \times 10^{18} \mathrm{H}$-atoms cm ${ }^{-2}$ (EBRG02), suggesting a much lower extinction, $A_{\mathrm{V}}<0.01$.

Using the spectral types and visual extinctions of the reference stars, we obtain the spectroscopic parallaxes given in

\footnotetext{
${ }^{1}$ Go to http://www.ctio.noao.edu/cfccd/cfccd.html for further information on this instrument.

${ }^{2}$ For a complete description of the CTIO 1.5-m Cassegrain Spectrograph go to

http://www. ctio.noao.edu/spectrographs/

$60 \mathrm{spec/manual/}$
}

the last column of Table 2. To determine these values, we used the Hipparcos calibration of the absolute magnitude for main sequence stars by Houk et al. (1997), and that for giant stars tabulated by Drilling \& Landolt (2000). For the astrometric solution discussed below, we assumed error bars of $\pm 25 \%$ on our spectroscopic parallaxes.

\subsection{The astrometric solution}

The data reduction process for deriving a parallax from FGS observations was identical to previous efforts, except for the fact that the astrometer has changed from FGS3 to FGS1R. FGS1R has been successfully calibrated (McArthur et al. 2002), and is the astrometric instrument of choice for current HST programs.

Solving the astrometric equations for the absolute rather than relative motion requires knowledge of the proper motions of the reference stars in addition to their parallaxes. We have used the USNO CCD Astrograph Catalogue (the "UCAC", Zacharias et al. 2000) in its on-line version $\left(V_{i z i e R^{3}}\right.$ catalogue $\mathrm{I} / 268$ ) to extract proper motions with errors for all six of our targets (Cols. 3 and 5 of Table 2).

With these input data, the astrometric equations (McArthur et al. 2001, Eqs. (1-4)) were solved simultaneously, using GaussFit (Jefferys et al. 1987) to minimize the $\chi^{2}$ values of the solution. Because of the large difference in $B-V$ between EX Hya and the reference stars, the lateral colour correction discussed in Benedict et al. (1999) is included. The final parallax of EX Hya is $\pi=15.50 \pm 0.29$ mas. The error of 0.29 mas exceeds that of previous parallax measurements with the HST FGS of the CVs TV Col and WZ Sge (McArthur et al. 2001; Harrison et al. 2003). The reason for the somewhat larger error for EX Hya is the lower number of epochs in our case and the noisy character of the reference frame.

As discussed by Lutz \& Kelker (1973), the nature of parallax measurements implies that the most probable true parallax is slightly smaller than the observed parallax of a star. The magnitude of the effect depends on the relative error of the observed parallax and on the model parameters describing the magnitude and space density distributions of the parent population (e.g. Hanson 1979). For the present observation of EX Hya, the probable value of the correction, $-0.04 \pm 0.02$ mas, is minute compared with the statistical error of the measurement of 0.29 mas and is subsequently neglected.

\section{Discussion}

The measured parallax corresponds to a distance of $d=64.5 \pm 1.2 \mathrm{pc}$ and confirms the earlier result of EBRG02 based on the somewhat uncertain interpretation of the ellipsoidal modulation of the secondary star. Combined with information on the masses of primary and secondary star, the now accurately known distance allows us to derive reliable values of the accretion luminosity and the accretion rate of EX Hya.

\footnotetext{
${ }^{3}$ Ochsenbein et al. (2000). http://vizier.u-strasbg.fr/ cgi-bin/VizieR
} 
Table 1. Photometric and spectroscopic data for the EX Hya reference frame.

\begin{tabular}{lcccccccc}
\hline \hline Star & $V$ & $B-V$ & $V-R$ & $V-I$ & $J-H$ & $H-K$ & $K$ & Spectral Type \\
\hline EX Hya & 13.26 & 0.13 & 0.25 & 0.79 & 0.34 & 0.21 & 11.75 & \\
EX-177 & 11.42 & 0.92 & 0.53 & 0.96 & 0.48 & 0.08 & 9.34 & K0.5V \\
EX-193 & 14.35 & 1.19 & 0.65 & 1.18 & 0.58 & 0.14 & 11.75 & K4V \\
EX-201 & 13.50 & 0.70 & 0.39 & 0.75 & 0.37 & 0.05 & 11.88 & G1.5V \\
EX-301 & 12.74 & 1.18 & 0.62 & 1.16 & 0.61 & 0.13 & 10.08 & K0III \\
EX-354 & 12.08 & 1.02 & 0.52 & 1.01 & 0.55 & 0.07 & 9.79 & G8III \\
\hline
\end{tabular}

Table 2. Positions, proper motions, visual extinctions and derived spectroscopic parallaxes for the EX Hya reference frame.

\begin{tabular}{lcccccc}
\hline \hline Star & $\alpha_{2000}$ & $P M_{\alpha}(\mathrm{mas} / \mathrm{yr})^{1}$ & $\delta_{2000}$ & $P M_{\delta}(\mathrm{mas} / \mathrm{yr})^{1}$ & $A_{\mathrm{V}}$ & $\pi(\mathrm{mas})^{2}$ \\
\hline EX Hya & 125224.4 & -127.3 & -291456.7 & +31.6 & $<0.01^{3}$ & \\
EX-177 & 125235.5 & -93.7 & -291259.5 & +11.1 & 0.20 & 8.54 \\
EX-193 & 125209.6 & -18.9 & -291336.6 & -7.8 & 0.21 & 3.94 \\
EX-201 & 125231.4 & -13.9 & -291401.8 & -25.3 & 0.20 & 1.66 \\
EX-301 & 125239.4 & +1.0 & -291458.2 & +5.7 & 0.49 & 0.47 \\
EX-354 & 125250.1 & -10.2 & -291538.9 & -1.2 & 0.21 & 0.56 \\
\hline
\end{tabular}

${ }^{1}$ Zacharias et al. (2000). ${ }^{2}$ Adopted error of $\pi$ is $25 \% .{ }^{3}$ Based on the Ly $\alpha$ absorption measurement of Eisenbart et al. (2002).

\subsection{Absolute magnitude of EX Hya}

The distance modulus of EX Hya is $m-M=4.05 \pm 0.04$. Our observed visual magnitude of EX Hya in quiescence, $m_{\mathrm{V}}=13.26$ (see Table 1), implies $M_{\mathrm{V}}=9.22$. EX Hya is slightly variable even in quiescence, however, and Hellier et al. (2000) quote a long-term orbital-mean quiescent magnitude $m_{\mathrm{V}}=13.0$, suggesting an average $M_{\mathrm{V}}$ in quiescence of 9.0. The absolute magnitude in outburst based on a mean peak magnitude of $m_{\mathrm{V}} \simeq 9.6$ (Hellier et al. 2000) is $M_{\mathrm{V}} \simeq 5$.6. This value has still to be corrected for inclination effects.

\subsection{Kinematic solution}

Recently, Belle et al. (2003) and Vande Putte et al. (2003) reported velocity amplitudes of the white dwarf and of the secondary star, $K_{1}=59.6 \pm 2.6 \mathrm{~km} \mathrm{~s}^{-1}$ and $K_{2}=360 \pm 35 \mathrm{~km} \mathrm{~s}^{-1}$, respectively. The inclination $i$ is defined by the length of the partial, but flat-bottomed eclipse of the X-ray emission from the lower magnetic pole, i.e., the one below the orbital plane (Beuermann \& Osborne 1988; Rosen et al. 1988), with a FWHM width of $157 \mathrm{~s}$ (Mukai et al. 1998). The kinematic solution yields $M_{1}=0.49 \pm 0.13 M_{\odot}, M_{2}=0.081 \pm 0.013 M_{\odot}$, and $i=76.6^{\circ} \pm 0.8^{\circ}$, where we have assumed that the lower pole is just visible through the inner hole in the accretion disk. For each additional $10^{8} \mathrm{~cm}$ required to be freely visible below the pole, the inclination decreases by $0.15^{\circ}$.

A white dwarf mass near $0.5 M_{\odot}$ was also advocated by Ezuka \& Ishida (1997), who obtained $M_{1}=0.50 \pm 0.08 M_{\odot}$ from X-ray line intensity ratios, and by Cropper et al. (1999), who derived $M_{1}=0.46 \pm 0.04 M_{\odot}$ from the X-ray continuum temperature. Compared with the kinematic solution, however, these indirect methods may be afflicted by systematic errors which are difficult to estimate. Presently, we allow, therefore, for the larger error in $M_{1}$ as given above.
As shown below, the remaining error in $K_{2}$ is the principal uncertainty in the discussion of the system parameters, the accretion rate, and the evolutionary status of EX Hya. For completeness, we note that the previous mass determinations of Hellier (1996) and Vande Putte et al. (2003) yield the same principal result, but were not based on the most recent $K_{1}, K_{2}$ combination. In particular, Hellier (1996) used the Smith et al. (1993) $K_{2}=356 \pm 4 \mathrm{~km} \mathrm{~s}^{-1}$ which has since been superseded by the reanalysis of the same data by Vande Putte et al. (2003) yielding the larger error quoted above.

\subsubsection{The primary star}

For $M_{1}=0.49 \pm 0.13 M_{\odot}$, Wood (1995) white dwarf models with a "thick" hydrogen envelope and an effective temperature of $10^{4} \mathrm{~K}$ predict $R_{1}=(1.02 \pm 0.15) \times 10^{9} \mathrm{~cm}$. Given the distance, we can compare this radius with that implied by the HST/GHRS UV flux and the mean effective temperature $T_{\text {eff,wd }}=25000 \pm 3000 \mathrm{~K}($ EBRG02, Belle et al. 2003) of the accreting white dwarf. For $d=64.5 \mathrm{pc}$, we obtain an effective radius $R_{1, \text { eff }} \simeq 6.0 \times 10^{8} \mathrm{~cm}$, which corresponds to $M_{1} \simeq 0.95 M_{\odot}$. Hence, either the white dwarf is much more massive than suggested by the kinematic solution or the UV emission originates only from the heated polar caps and the underlying white dwarf is much cooler. Below, we shall exclude $M_{1} \gtrsim 0.8 M_{\odot}$ and conclude, therefore, that the second possibility must hold. Large warm spots seem to be the rule in pole-accreting white dwarfs in AM Her stars and probably exist also in IPs (e.g., Gänsicke et al. 1995; Gänsicke et al. 1998; Gänsicke 2000). This is consistent with the fact that the typical white dwarf temperature expected from compressional heating in short-period CVs is $10000-20000 \mathrm{~K}$ (Townsley \& Bildsten 2002; Gänsicke 2000; Gänsicke et al. 2000; Szkody 2002). Modelling the HST/GHRS spectrum of EX Hya with a 
polar cap of $25000 \mathrm{~K}$ and a cooler underlying white dwarf of $10^{9} \mathrm{~cm}$ radius at the measured distance of EX Hya yields an approximate upper limit to the effective temperature of the latter of $17000 \mathrm{~K}$.

\subsubsection{The secondary star}

The low kinematic mass of the secondary star may surprise at first glance. Such a low mass, however, is in line with the secondary masses in Z Cha (Wade \& Horne 1988), HT Cas (Horne et al. 1991), and OY Car (Wood et al. 1989). These results for short-period CVs suggest that with decreasing orbital period the radii of the Roche-lobe filling secondaries exceed those of ZAMS objects (Baraffe et al. 1998) and that the secondaries are slightly out of thermal equilibrium. The secondary in EX Hya falls in line with this trend. For $M_{2}=0.081 \pm 0.013 M_{\odot}$, its Roche radius is $R_{2, \mathrm{R}}=(9.58 \pm 0.50) \times 10^{9} \mathrm{~cm}$ while a Rochelobe filling ZAMS-star would have a radius smaller by $15-23 \%$ (Baraffe et al. 1998; Renvoizé et al. 2002).

Given the distance, the radius of the secondary can also be estimated from its luminosity and effective temperature. At $d=64.5 \mathrm{pc}$, the $K$-band magnitude of the secondary star $K \simeq 12.4 \pm 0.3$ (EBRG02) implies an absolute magnitude $M_{\mathrm{K}}=8.4 \pm 0.3$. Using the stellar models with solar metallicity of Baraffe et al. (1998), we convert $M_{\mathrm{K}}$ to a bolometic luminosity $L_{2} \simeq 0.0024 \pm 0.0006 L_{\odot}$. With an effective temperature for the dM4 \pm 1 secondary in EX Hya (EBRG02) of $T_{\text {eff }} \simeq 3200-3300 \mathrm{~K}$ (Leggett et al. 1996, 2000), we then obtain a radius $R_{2}=(10.6 \pm 1.6) \times 10^{9} \mathrm{~cm}$, consistent with the kinematically determined Roche-lobe radius.

In summary, the kinematic solution for EX Hya is consistent with the picture which evolves from studies of other short-period CVs. While the spectral type of the secondary in EX Hya is as expected for a Roche-lobe filling main sequence star in a CV of the same orbital period (Beuermann et al. 1998), the kinematic solution suggests that the secondary is undermassive, overluminous and expanded over a ZAMS object.

\subsection{Quiescent accretion luminosity}

The mean orbital observed flux of EX Hya integrated over all wavelengths from the infrared to the hard X-ray regime is $(6.0 \pm 0.6) \times 10^{-10} \mathrm{erg} \mathrm{cm}^{-2} \mathrm{~s}^{-1}$ (EBRG02, their Table 4). This value includes the Fe $\mathrm{L}$ excess flux around $1 \mathrm{keV}$ (Mukai 2001), an estimate of the hard X-ray flux beyond $20 \mathrm{keV}$, and an estimate of the (small) XUV flux shortward of the Lyman edge. It also includes the flux received from the white dwarf, but excludes that from the secondary star. According to EBRG02, about $1.8 \times 10^{-10} \mathrm{erg} \mathrm{cm}^{-2} \mathrm{~s}^{-1}$ is from to the white dwarf. As noted above, we attribute much of this flux to reprocessed radiation from its irradiated pole caps. In order to obtain the quiescent accretion-induced flux, the fraction originating from the unheated white dwarf photosphere has to be subtracted. For a range of effective temperatures of 10000 $17000 \mathrm{~K}$ (see above), an $0.5 M_{\odot}$ white dwarf contributes $(0.7 \pm$ $0.5) \times 10^{-10} \mathrm{erg} \mathrm{cm}^{-2} \mathrm{~s}^{-1}$ to the wavelength-integrated flux. Subtracting this contribution, we obtain the accretion-induced
Table 3. Comparion of luminosity derived and theoretically predicted accretion rates. Column (2) is the effective exponent of the mass radius relation of the secondary star, Cols. (2) and (3) are for the nominal kinematic solution, $M_{1}, M_{2}=0.49,0.081 M_{\odot}$, and Cols. (4) and (5) for the $+1 \sigma$ limit of $K_{2}, M_{1}, M_{2}=0.62,0.094 M_{\odot}$. Accretion rates are in $M_{\odot} \mathrm{yr}^{-1}$, inner disk radii $r_{\mathrm{i}, 9}$ are in units of $10^{9} \mathrm{~cm}$.

\begin{tabular}{|c|c|c|c|c|c|}
\hline Model & $\begin{array}{c}(1) \\
\zeta\end{array}$ & $\begin{array}{c}(2) \\
\lg \dot{M}_{1}\end{array}$ & $\begin{array}{l}(3) \\
r_{i, 9}\end{array}$ & $\begin{array}{c}(4) \\
\lg \dot{M}_{1}\end{array}$ & $\begin{array}{l}(5) \\
r_{\mathrm{i}, 9}\end{array}$ \\
\hline \multicolumn{6}{|c|}{ (a) Luminosity-derived accretion rate } \\
\hline & & $\begin{array}{r}-10.17 \\
\pm 0.10\end{array}$ & & $\begin{array}{r}-10.34 \\
\pm 0.10\end{array}$ & \\
\hline \multicolumn{6}{|c|}{ (b) Model accretion rates and inner disk radii } \\
\hline $\begin{array}{l}r_{\mathrm{i}, \max }, \dot{M}_{\min } \\
r_{\mathrm{i}, \min }, \dot{M}_{\max }\end{array}$ & & $\begin{array}{l}-10.54 \\
-10.13\end{array}$ & $\begin{array}{r}27.4 \\
4.4\end{array}$ & $\begin{array}{l}-10.65 \\
-10.19\end{array}$ & $\begin{array}{r}30.0 \\
4.0\end{array}$ \\
\hline gravitational only & $\begin{array}{r}0.85 \\
0.50 \\
-0.20\end{array}$ & $\begin{array}{l}-10.93 \\
-10.86 \\
-10.65\end{array}$ & & $\begin{array}{l}-10.73 \\
-10.66 \\
-10.46\end{array}$ & \\
\hline grav + spin-up & $\begin{array}{r}0.85 \\
0.50 \\
-0.20\end{array}$ & $\begin{array}{l}-10.45 \\
-10.37 \\
-10.17\end{array}$ & $\begin{array}{r}19.6 \\
13.9 \\
5.4\end{array}$ & $\begin{array}{l}-10.43 \\
-10.36 \\
-10.16\end{array}$ & $\begin{array}{r}12.2 \\
8.6 \\
3.4\end{array}$ \\
\hline $3 \times$ grav + spin-up & $\begin{array}{r}0.85 \\
0.50 \\
-0.20\end{array}$ & $\begin{array}{r}-10.23 \\
-10.16 \\
-9.95\end{array}$ & $\begin{array}{l}7.2 \\
5.1 \\
2.0\end{array}$ & $\begin{array}{c}-10.13 \\
-10.06 \\
-9.86\end{array}$ & $\begin{array}{l}3.0 \\
2.2 \\
0.9\end{array}$ \\
\hline
\end{tabular}

flux during quiescence, $f_{\mathrm{q}}=(5.3 \pm 0.8) \times 10^{-10} \mathrm{erg} \mathrm{cm}^{-2} \mathrm{~s}^{-1}$. The corresponding quiescent accretion luminosity of EX Hya is $L_{\mathrm{q}}=4 \pi d^{2} f_{\mathrm{q}}=(2.6 \pm 0.6) \times 10^{32} \mathrm{erg} \mathrm{s}^{-1}$, where we have quadratically added an additional error of $20 \%$ for basing $L_{\mathrm{q}}$ on the mean orbital flux instead of on the $4 \pi$-averaged flux.

\subsection{Accretion rate}

\subsubsection{Quiescence}

The observationally determined accretion rate in quiescence, $\dot{M}_{\mathrm{q}}$, is related to the luminosity by $L_{\mathrm{q}} \simeq G M_{1} \dot{M}_{\mathrm{q}} / R_{1}$. We take $M_{1}$ for the moment as a free parameter and approximate the Wood (1995) radii of white dwarfs with a thick hydrogen envelope as $R_{1} \simeq 10.0 \times 10^{8}\left(M_{1} / 0.50 M_{\odot}\right)^{-0.61} \mathrm{~cm}$, valid for masses between 0.40 and $0.65 M_{\odot}$. The luminosity can then be converted to a quiescent accretion rate $\dot{M}_{\mathrm{q}}=(6.2 \pm 1.4) \times$ $10^{-11}\left(M_{1} / 0.5 M_{\odot}\right)^{-1.61} M_{\odot} \mathrm{yr}^{-1}$. This value is valid as an average over the last 30 years over which the quiescent magnitude of EX Hya has stayed approximately constant.

\subsubsection{Time-average including dwarf nova outbursts}

$\dot{M}_{\mathrm{q}}$ has still to be corrected for the effect of the rare dwarf nova outbursts, in which the visual magnitude of EX Hya rises from $13 \mathrm{mag}$ to about $10 \mathrm{mag}$ for 1-2 days. Hellier et al. (2000) assumed that the mass transfer rate scales as the optical brightness and estimated that the outbursts typically involve $10^{22} \mathrm{~g}$. Using his gap-corrected outburst recurrence rate of $1.5 \mathrm{yrs}$ suggests that the time-averaged accretion rate $\dot{M}_{1, \text { av }} \simeq 1.05 \times \dot{M}_{\mathrm{q}}=$ $(6.5 \pm 1.5) \times 10^{-11}\left(M_{1} / 0.5 M_{\odot}\right)^{-1.61} M_{\odot} \mathrm{yr}^{-1}$. 


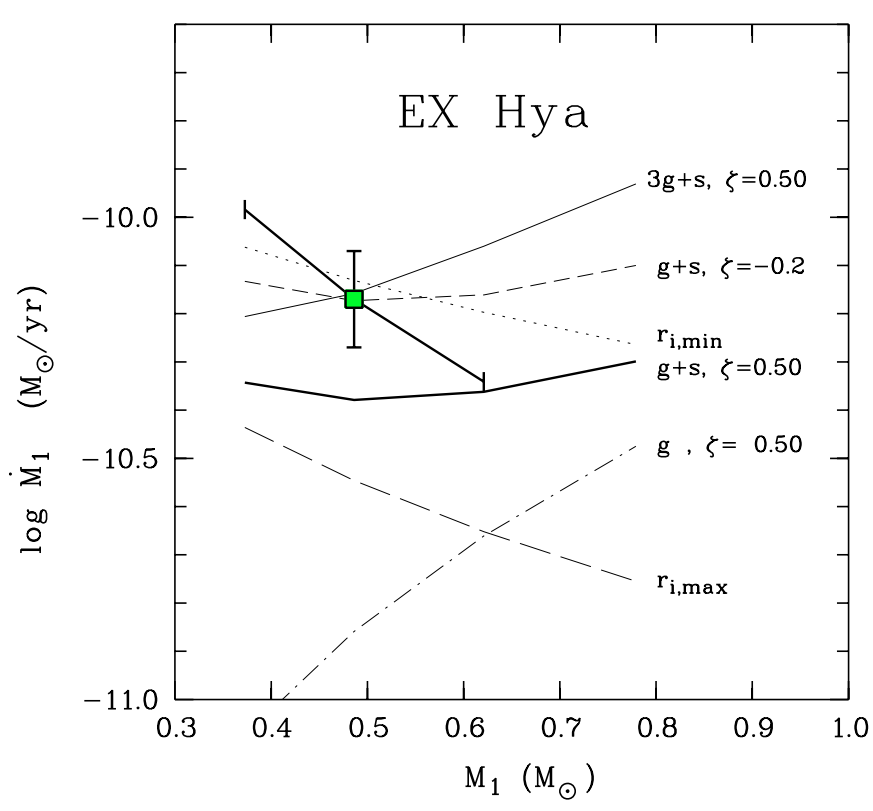

Fig. 1. Accretion rate as a function of $M_{1}$ for the spin-up rate of Hellier \& Sproats (1992) and different assumptions on the driving mechanism, gravitational radiation only $(\mathrm{g})$, gravitational radiation plus spin-up $(\mathrm{g}+\mathrm{s})$, and a systemic angular momentum loss of $3 \times$ the gravitational rate plus spin-up $(3 g+s)$. Accretion from the corotation radius defines a mininal accretion rate (long-dashed curve, $r_{\mathrm{i}}=r_{\mathrm{co}}$ ). The luminosityderived accretion rate of EX Hya is shown as the solid square with error bars (see text).

\subsection{Theoretical accretion rate}

The accretion rate is physically related to the spin-up rate of the white dwarf which has been observed over a time span of 30 years, $-P_{1} / \dot{P}_{1}=(3.8 \pm 0.2) \times 10^{6}$ yrs $($ Hellier \& Sproats 1992). Equivalently, $\dot{P}_{1}=-(3.4 \pm 0.2) \times 10^{-11}$ and $\dot{\Omega}_{1}=(1.32 \pm$ $0.07) \times 10^{-17} \mathrm{~s}^{-2}$.

The relation between spin-up of the white dwarf and accretion rate has been extensively discussed by Ritter (1985) for the case of conservative mass transfer, i.e., $\dot{M}_{1}=-\beta \dot{M}_{2}$ with $\beta=1$. The alternative extreme of $\beta=0$ applies to the case that the accreted mass is shed off again from the white dwarf. Non-conservative mass transfer was treated by Rappaport et al. (1983) and considered in recent evolutionary calculations (e.g., Kolb \& Baraffe 1999; Howell et al. 2001). We adopt the conservative case here because (i) a wind is unlikely to flow off from the magnetic white dwarf, (ii) propeller action is unlikely to be very efficient given the slow rotation of the white dwarf, and (iii) the relation between accretion and spin-up considered here is that over the last 30 years and not a secular one, which obviates the need to account for mass and angular momentum loss in nova eruptions. The difference in the predicted mass transfer rates for EX Hya between the $\beta=1$ and $\beta=0$ cases amount to only $\Delta\left(\log \dot{M}_{2}\right) \simeq 0.050$ because the specific orbital angular momentum of the white dwarf is small.

Using Ritter's (1985) Eq. (1) or (A19), we calculate the accretion rate $\dot{M}_{1}$ driven by a systemic angular momentum loss rate $\dot{J}_{\text {sys }}$, for given $M_{1}, M_{2}, \dot{\Omega}_{1}$, and an effective mass radius exponent of the secondary star, $\zeta=\mathrm{d} \ln R_{2} / \mathrm{d} \ln M_{2}$ (with $\zeta \equiv \alpha_{2}$ in Ritter 1985). The transfer rate $\dot{M}_{1}$ increases with decreasing
Table 4. System parameters of EX Hydrae (see text).

\begin{tabular}{lll}
\hline \hline Parameter & Value or range & Ref. \\
\hline (a) Observed parameters, literature: & \\
Orbital period $P_{\text {orb }}$ & $5895.4 \mathrm{~s}$ & $(1)$ \\
Spin period $P_{1}$ & $4021.6 \mathrm{~s}$ & $(1)$ \\
Spin-up rate $P_{1} / \dot{P}_{1}$ & $(3.8 \pm 0.2) \times 10^{6} \mathrm{yrs}$ & $(1)$ \\
Velocity amplitude $K_{1}$ & $59.6 \pm 2.6 \mathrm{~km} \mathrm{~s}^{-1}$ & $(2)$ \\
Velocity amplitude $K_{2}$ & $360 \pm 35 \mathrm{~km} \mathrm{~s}^{-1}$ & $(3)$ \\
Width of X-ray eclipse & $157 \mathrm{~s}$ & (4)
\end{tabular}

(b) Kinematic solution based on literature values:

White dwarf mass $M_{1} \quad 0.49 \pm 0.13 M_{\odot}$

Secondary mass $M_{2} \quad 0.081 \pm 0.013 M_{\odot}$

Inclination $i \quad 76.6^{\circ} \pm 1.0^{\circ}$

(c) Observed parameters, this work:

HST FGS Parallax $\pi \quad 15.50 \pm 0.29$ mas

Distance $d \quad 64.5 \pm 1.2 \mathrm{pc}$

Accretion rate $\dot{M}_{1 \text {,av }} \quad(6.6 \pm 1.5) \times 10^{-11} \times$

$$
\left(M_{1} / 0.5 M_{\odot}\right)^{-1.61} M_{\odot} \mathrm{yr}^{-1}
$$

(d) Comparison with theory, this work:

White dwarf mass $M_{1} \quad 0.40-0.70 M_{\odot}$

Secondary mass $M_{2} \quad 0.07-0.10 M_{\odot}$

Implied range of $K_{2} \quad 335-413 \mathrm{~km} \mathrm{~s}^{-1}$

White dwarf radius $R_{1} \quad(0.017-0.012) R_{\odot}$

Roche radius $R_{2} \quad(0.132-0.148) R_{\odot}$

Inclination $i \quad 76.0^{\circ}-77.6^{\circ}$

Inner edge of disk $r_{\mathrm{i}} \quad(5-9) \times 10^{9} \mathrm{~cm}$

Accretion rate $\dot{M}_{1} \quad(8-4) \times 10^{-11} M_{\odot} \mathrm{yr}^{-1}$

(1) Hellier \& Sproats (1992), (2) Belle et al. (2003), (3) Vande Putte et al. (2003), (4) Mukai et al. (1998).

$\zeta$. Equilibrium models of low mass main sequence stars yield $\zeta \simeq 0.85$ (Baraffe et al. 1998). As mentioned above, however, the observed masses of Z Cha, HT Cas, OY Car, and WZ Sge and the requirement that the stars fill their Roche lobes suggest some bloating beyond that caused already by deformation of the star in the Roche potential (Renvoizé et al. 2002), consistent with $\zeta \sim 0.5$ and a mild loss of thermal equilibrium. The minimum value which $\zeta$ can assume in fully convective stars is near the adiabatic limit, $\zeta_{\text {ad }} \simeq-1 / 3$, and may be realized after turnon of mass transfer (d'Antona et al. 1988). An additional model parameter is the efficiency of the driving mechanism. We chose two cases: (i) gravitational radiation only, supplemented by the observed spin-up and (ii) the same plus an unspecified additional mechanism twice as effective as gravitaional radiation. The resulting $\dot{M}_{1}$ for these models are listed in Table 3 for two $M_{1}, M_{2}$-combinations, the nominal kinematic solution and the $+1 \sigma$ value of $K_{2}$ (Vande Putte et al. 2003), i.e., $M_{1}=0.49 M_{\odot}$ and $M_{1}=0.62 M_{\odot}$, respectively. For completeness, we include the "gravitational only" case (without spin-up).

\subsection{EX Hya as an intermediate polar}

Figure 1 shows the luminosity-derived $M_{1}$-dependent accretion rate by the filled square, with the inclined "horizontal" error bar indicating the $\pm 1 \sigma$-range of $M_{1}$. Also shown are the accretion rates for selected models and for a larger range of $M_{1}$, with " $\mathrm{g}$ " 
referring to angular momentum loss by gravitational radiation and and "s" to the spin-up contribution. Several interesting conclusions can be drawn from a comparison of these rates. While the angular momentum drain by gravitational radiation can not be avoided, the spin-up observed over 30 years signifies an additional transfer of orbital angular momentum into the spin of the white dwarf which increases $\dot{M}_{1}$. The combined effect is described by the $g+s$ model (Ritter 1985, his Eq. (1), see also the details in the Appendix to his paper). Since the secondary in EX Hya is slighly expanded over a main sequence object, we chose an effective $\zeta=0.50$. This case represents an approximate lower limit to $\dot{M}_{1}$ for the current state of EX Hya (thick solid curve in Fig. 1). $\dot{M}_{1}$ for $\zeta \simeq 0.85$ would be lower by only $16 \%\left(\Delta\left(\log \dot{M}_{2}\right) \simeq 0.075\right.$, see Table 3$)$.

Independent information on $\dot{M}_{1}$ can be gathered from equating the time derivative of the white dwarf's spin angular momentum to the torque exerted by matter which couples to the white dwarf's magnetosphere at a lever arm $r_{\mathrm{i}}$, i.e., $J_{\mathrm{s}, 1}=\dot{M}_{1} r_{\mathrm{i}} v_{\mathrm{K}}\left(r_{\mathrm{i}}\right)$, where $v_{\mathrm{K}}\left(r_{\mathrm{i}}\right)$ is the Keplerian velocity at $r_{\mathrm{i}}$ and $\dot{J}_{\mathrm{s}, 1}$ depends on the spin-up rate $\dot{\Omega}_{1}$ (and the structural change of the white dwarf associated with $\dot{M}_{1}$ ) (see Eqs. (6), (7) and (A15) of Ritter 1985). For given $\dot{\Omega}_{1}$ and $\dot{M}_{1}$, the equality defines the radial lever arm $r_{i}$, which we identify with the inner edge of the accretion disk or, vice versa, chosing limits on the lever arm defines limiting values for $\dot{M}_{1}$. To be sure, this is strictly correct only if the inner disk is in Keplerian motion and coupling occurs only at $r_{\mathrm{i}}$ (and not also further out), which may not be the case (e.g., King \& Wynn 1999). We do not further consider this question here and only note that in these more general cases the actual inner edge of the circulating material, loosely referred to as disk here, would be located inside the sodefined $r_{\mathrm{i}}$. Two conditions thus restrict the permitted range of $\dot{M}_{1}$ independent of all other arguments. A lower limit $\dot{M}_{\min }$ is given by $r_{\mathrm{i}, \max }=r_{\mathrm{L} 1}$, the distance to the $\mathrm{L}_{1}$ point (long dashed curve in Fig. 1). Correspondingly, an upper limit $\dot{M}_{\max }$ is given by $r_{\mathrm{i}, \mathrm{min}}=R_{1} / \cos i$, the inner disk radius which just allows the lower pole of the white dwarf to be viewed through the inner hole in the disk (dotted curve in Fig. 1). This free view is required by the partial, yet flat-bottomed X-ray eclipse (Mukai et al. 1998, see also Beuermann \& Osborne 1988; Rosen et al. 1988). If one requires the emission at a distance larger than $R_{1}$ below the orbital plane to be visible through the hole, $r_{\mathrm{i}}$ has to be correspondingly larger, which would move the dotted curve in Fig. 1 further downward. The permitted range of $r_{\mathrm{i}}$ (see Table 4) is consistent with that deduced spectroscopically by Hellier et al. (1987).

With the above conditions, the range permitted for $\dot{M}_{1}$ in Fig. 1 is restricted to below the dotted and above the thick solid curve. Interestingly, this range vanishes for $M_{1} \gtrsim 0.8 M_{\odot}$ and, hence, excludes a white dwarf mass above this limit. Gratifyingly, $\dot{M}_{1, \text { av }}$ for the range of $M_{1}$ as given by the kinematic solution is entirely consistent with this permitted area in the $\dot{M}_{1}, M_{1}$-plane. Without knowledge of the kinematic masses, the permitted area alone restricts $M_{1}$ to stay between about $0.40 M_{\odot}$ and $0.70 M_{\odot}$. This mass range corresponds to $K_{2}=$ $335-415 \mathrm{~km} \mathrm{~s}^{-1}$ in excellent agreement with Vande Putte's (2003) $K_{2}=360 \pm 35 \mathrm{~km} \mathrm{~s}^{-1}$. Table 4 summarizes our results.
The error in $\dot{M}_{1}$ could be reduced to that in the bolometric flux if a precise measurement of $M_{1}$ or $R_{1}$ would be available.

Finally, we comment on the evolutionary state of EX Hya and the properties of the mass-losing secondary star. For $M_{1} \simeq$ $0.6-0.7 M_{\odot}$, the implied $\dot{M}_{1, \text { av }} \simeq 4 \times 10^{-} 11 M_{\odot} \mathrm{yr}^{-1}$ is entirely consistent with near equilibrium mass transfer under the action of gravitational radiation and spin-up (model $\mathrm{g}+\mathrm{s}$ with $\zeta \simeq 0.5-0.9$ ). For $M_{1} \simeq 0.4-0.5 M_{\odot}$, near the best-fit kinematic and X-ray-derived primary masses, the higher transfer rate $\dot{M}_{1 \text {,av }} \simeq 8 \times 10^{-11} M_{\odot} \mathrm{yr}^{-1}$ can be provided in two scenarios: (i) A more efficient angular momentum loss process is at work, which we describe as gravitational radiation plus an additional loss process which is about twice as efficient as the former (model $3 \mathrm{~g}+\mathrm{s}$ with $\zeta \simeq 0.5-0.9$ in Table 3 and Fig. 1 ). Such process has been invoked in numerous investigations dealing with open questions in CV evolution (e.g. Patterson 1998; Kolb \& Baraffe 1999; Schwope et al 2002). (ii) In the second scenario, only gravitational radiation is effective, but the secondary has lost thermal equilibrium after the sudden turn-on of mass transfer causing its effective mass radius exponent $\zeta$ to drop to a value near the adiabatic one, $\zeta_{\text {ad }} \simeq-1 / 3$, (model $g+s$ with an arbitrarily chosen $\zeta \simeq-0.2$ ). In this case, the orbital period would currently be stagnating or increasing, $\dot{M}_{1}$ would exceed the equilibrium value by about a factor of three, and the object would be located near the "tip of the flagpole", the flag being the loop described by the evolving CV in the $\dot{M}_{1}, P_{\text {orb- }}$ plane (d'Antona et al. 1988; Kolb \& Baraffe 1999). It is noteworthy that turn-on would take a couple of million years which agrees with the time span over which the current spin-up rate in EX Hya might have been sustained if it started from a synchronized white dwarf. We can not presently distinguish between the scenarios (i) and (ii). The latter possibility might hold for EX Hya as a single object, but not for short-period CVs in general. A more precise primary mass would allow to place tighter limits on the evolutionary state of EX Hya.

\section{Conclusion}

Our high precision astrometric parallax has led to an internally consistent picture of EX Hya as an intermediate polar. With a distance of $d=64.5 \pm 1.2 \mathrm{pc}$, the luminosity-derived, time-averaged accretion rate of the white dwarf in EX Hya is $\dot{M}_{1, \text { av }}=(6.5 \pm 1.5) \times 10^{-11}\left(M_{1} / 0.5 M_{\odot}\right)^{-1.61} M_{\odot} \mathrm{yr}^{-1}$, valid over about the last 30 years. The largest uncertainty in pinning down $\dot{M}_{1, \text { av }}$ is still the poorly known primary mass $M_{1}$. The kinematic solution, the interpretation of X-ray observations (given a somewhat lower weight), and a range of other indirect arguments lead to a permitted range of $M_{1}=0.40-0.70 M_{\odot}$, with a preference for masses around $0.5 M_{\odot}$. Only for $M_{1}=$ 0.6-0.7 $M_{\odot}$, would the time-averaged accretion rate $\dot{M}_{1, \mathrm{av}}=$ $4 \times 10^{-11} M_{\odot} \mathrm{yr}^{-1}$ coincide with that expected from quasiequilibrium mass transfer driven by gravitational radiation plus the transfer of orbital into spin angular momentum, fixed by the observed spin-up time scale of $(3.8 \pm 0.2) \times 10^{6} \mathrm{yrs}$ (Hellier $\&$ Sproats 1992). If, on the other hand, $M_{1}=0.4-0.5 M_{\odot}$, the implied higher accretion rate $\dot{M}_{1, \text { av }}=8 \times 10^{-11} M_{\odot} \mathrm{yr}^{-1}$ requires either an additional angular momentum loss process or the loss of thermal equilibrium of the secondary star. In any 
case, a more precise value of $M_{1}$ would be an invaluable ingredient for a more detailed discussion of the evolutionary status of EX Hya.

Spectroscopically, the secondary looks like a main sequence star as expected in a CV with $98 \mathrm{~min}$ orbital period $\left(M_{2} \simeq 0.13 M_{\odot}\right)$, the permitted range of $M_{2}=0.07-0.10 M_{\odot}$ indicates, however, that it is undermassive, overluminous, and expanded over a main ZAMS object of the same mass.

Our results confirm some of the basic concepts developed for CVs over the last decades and demonstrates that accurate measurements of parallax and velocity amplitudes can provide detailed information on the evolutionary status of individual CVs.

Acknowledgements. This research was supported in Germany by DLR/BMFT grant 50 OR 99031 . In the United States, partial support for TEH, BEM, and GFB for proposal \#9230 was provided by NASA through a grant from the Space Telescope Science Institute, which is operated by the Association of Universities for Research in Astronomy, Inc., under NASA contract NAS 5-26555. In the UK, BTG was supported by a PPARC Advanced Fellowship. This research has made use of the NASA/ IPAC Infrared Science Archive, which is operated by the Jet Propulsion Laboratory, California Institute of Technology, under contract with the National Aeronautics and Space Administration. This publication also makes use of data products from the Two Micron All Sky Survey, which is a joint project of the University of Massachusetts and the Infrared Processing and Analysis Center/California Institute of Technology, funded by the National Aeronautics and Space Administration and the National Science Foundation. We also would like to thank B. Skiff for pointing us to the UCAC catalogue.

\section{References}

Baraffe, I., Chabrier, G., Allard, F., \& Hauschildt, P. 1998, A\&A, 337, 403

Belle, K. E., Howell, S., Sion, E. M., Long, K. M., \& Szkody, P. 2003, ApJ, 587, 373

Benedict, G. F., McArthur, B. E., Chappell, D. W., et al. 1999, AJ, 118,1086

Berriman, G., Szkody, P., \& Capps, R. W. 1985, MNRAS, 217, 327

Bessell, M. S., \& Brett, J. M. 1988, PASP, 100, 1134

Beuermann, K., \& Osborne, J. 1988, A\&A, 189, 128

Beuermann, K., Baraffe, I., Kolb, U., \& Weichhold, M. 1998, A\&A, 339,518

Carpenter, J. M. 2001, AJ, 121, 2851

Cropper, M., Wu, K., \& Kocabiyik, A. 1999, MNRAS, 306, 684

d'Antona, F., Mazzitelli, I., \& Ritter, H. 1989, A\&A, 225, 391

Drilling, J. S., \& Landolt, A. U. 2000, in Astrophysical Quantities, ed. A. Cox (New York: Springer-Verlag), 381

Eisenbart, S., Beuermann, K., Reinsch, K., \& Gänsicke, B. T. 2002, A\&A, 382, 984 (EBRG02)

Ezuka, H., \& Ishida, M. 1999, ApJS, 120, 277

Gänsicke, B. T., Beuermann, K., \& de Martino, D. 1995, A\&A, 303, 127

Gänsicke, B. T., Hoard, D. W., Beuermann, K., Sion, E. M., \& Szkody, P. 1998, A\&A, 338, 933

Gänsicke, B. T. 2000, Rev. Mod. Astr., 13, 151

Gänsicke, B. T., Beuermann, K., de Martino, D., \& Thomas, H.-C. 2000, A\&A, 354, 605
Hanson, R. B. 1979, MNRAS, 186, 875

Harrison, T. E., McNamara, B. J., Szkody, P., et al. 1999, ApJ, 515, L93

Harrison, T. E., McNamara, B. J., Szkody, P., \& Gilliland R. 2000, AJ, 120, 2649

Harrison, T. E., Johnson, J. J., McArthur, B. E., et al. 2003, AJ, in press

Hellier, C. 1996, in Cataclymsic Variables and Related Objects, ed. A. Evans, \& J. H. Wood (Kluwer), 143

Hellier, C., Mason, K. O., Rosen, S. R., \& Córdova, F. A. 1987, MNRAS, 228, 463

Hellier, C., \& Sproats, L. N. 1992, IBVS No. 3724

Hellier, C., Kemp, J., Naylor, T., et al. 2000, MNRAS, 313, 703

Horne, K., Wood, J. H., \& Stiening, R. F. 1991, ApJ, 378, 271

Houk, N., Swift, C. M., Murray, C. A., Penston, M. J., \& Binney, J. J. 1997, in Hipparcos-Venice, ed. M. Perryman (Noordwijk: ESA), p. 279

Howell, S. B., Nelson, L., \& Rappaport, S. 2001, ApJ, 550, 897

Jefferys, W., Fitzpatrick, J., \& McArthur, B. 1987, Celest. Mech., 41, 39

King, A. R., \& Wynn, G. A. 1999, MNRAS, 310, 203

Kolb, U., \& Baraffe, I. 1999, MNRAS, 309, 1034

Leggett, S. K., Allard, F., Berriman, G., Dahn, C. C., \& Hauschildt, P. H. 1996, ApJS, 104, 117

Leggett, S. K., Allard, F., Dahn, C. C., et al. 2000, ApJ, 535, 965

Lutz, T. E., \& Kelker, D. H. 1973, PASP, 85, 573

McArthur, B. E., Benedict, G. F., Lee J., et al. 1999, ApJ, 520, L59

McArthur, B. E., Benedict, G. F., Lee J., et al. 2001, ApJ, 560, 907

McArthur, B. E., Benedict, G. F., Jefferys, W. H., \& Nelan, E, in Proc. of the 2002 HST Calibration Workshop, ed. S. Arribas, A. Koekemoer, \& B. Whitmore, ASP Conf. Ser., 373

Mukai, K., Ishida, M., \& Osborne, J. P. 1998, ASP Conf. Ser., 137, 554

Mukai, K. 2001, COSPAR Sci. Ass. Warzaw, Adv. Space Res., in press Ochsenbein, F., Bauer, P., \& Marcout, J. 2000, A\&AS, 143, 221

Patterson, J. 1998, PASP, 110, 1132

Rappaport, S., Verbunt, F., \& Joss, P. C. 1983, ApJ, 275, 713

Reike, G. H., \& Lebofsky, M. J. 1985, ApJ, 288, 618

Renvoizé, V., Baraffe, I., Kolb, U., \& Ritter, H. 2002, A\&A, 389, 485

Ritter, H. 1985, A\&A, 148, 207

Rosen, S. R., Mason, K. O., \& Córdova, F. A. 1988, MNRAS, 231, 549

Schwope, A. D., Hambaryan, V., Schwarz, R., Kanbach, G., \& Gänsicke, B. T. 2002, A\&A, 392, 541

Smith, R. C., Collier-Cameron, A., \& Tucknott, D. S. 1993, in Cataclysmic Variables and Related Physics, ed. O. Regev, \& G. Shaviv, Ann. Israel Phys. Soc., 10, 70

Szkody, P., Sion, E. M., Gänsicke, B. T., \& Howell, S. B. 2002, ASP Conf. Ser., 261, 21

Townsley D. M., \& Bildsten, L. 2002, ApJ, 565, L35

Vande Putte, D., Smith, R. C., Hawkins, N. A., \& Martin, J. S. 2003, MNRAS, 342, 151

Wade, R. A., \& Horne, K. 1988, ApJ, 324, 411

Warner, B. 1987, MNRAS, 227, 23

Wood, M. 1995, in Lecture Notes in Physics, ed. D. Koester, \& K. Werner, LNP 443, 41

Wood, J. H., Horne, K., Berriman, G., \& Wade, R. 1989, ApJ, 341, 974

Zacharias, N., Urban, S. E., Zacharias, M. I., et al. 2000, AJ, 120, 2131 